\title{
Theoretical Analysis of Control Properties for the Brushless Doubly Fed Reluctance Machine
}

\author{
Robert E. Betz, Member, IEEE, and Milutin G. Jovanović, Member, IEEE
}

\begin{abstract}
The brushless doubly fed induction machine (BDFIM) has been extensively researched for approximately 30 years, but a related machine [the brushless doubly fed reluctance machine (BDFRM)], has not. This was mainly due to the fact that reluctance rotor designs were not capable of generating saliency ratios large enough to make the BDFRM competitive with other machines. However, recent developments in reluctance rotors, spurred on by research into synchronous reluctance machines, has resulted in high saliency ratio rotors that are economic to build. This, together with the promise of higher efficiency and simpler control compared to the BDFIM, means that further investigation of the BDFRM is warranted. A relatively limited amount of work to date has been published on the BDFRM. This paper attempts to fill this void by presenting a theoretical analysis of some of the important control properties of the ideal BDFRM.
\end{abstract}

Index Terms-Brushless doubly fed machine, electric machine control, reluctance machine, self-cascade machine.

\section{INTRODUCTION}

$\mathbf{T}$ HE BRUSHLESS doubly fed reluctance machine (BDFRM) belongs to a group of interesting machines that include the classical cascaded induction machine (CIM), the traditional double-fed slip ring induction machine (DFSRIM), and the brushless doubly fed induction machine (BDFIM).

The CIM, which forms the philosophical basis for the BDFRM and BDFIM, is a very old machine. Papers outlining its basic principles appeared at the beginning of the last century [1]-[3]. The next step in the development of this machine did not occur until approximately 50 years later [4]-[6]. Essentially, the two-machine cascade system was collapsed into a single frame. The two variants developed (the BDFIM and the BDFRM) used the same stator but the BDFRM replaced a special cage rotor of the BDFIM with a reluctance one.

In the last 10 years, there has been a renewed interest in the BDFIM and the BDFRM due to the slip recovery nature of their operation. This means that if the speed range of operation is limited to about a "synchronous" speed, then the inverter can be fractionally rated. Even though the machine needs to be larger for a given torque output, this fact means that the total system cost may be lower.

In many respects, the BDFRM and the BDFIM are very similar; however, the BDFRM has the potential for greater effi-

Manuscript received May 23, 2000; revised February 24, 2002.

R. E. Betz is with the Department of Electrical and Computer Engineering, University of Newcastle, Callaghan, NSW, 2308, Australia (e-mail: reb@ecemail.newcastle.edu.au).

M. G. Jovanović is with the School of Engineering, University of Northumbria at Newcastle, Newcastle upon Tyne NE1 8ST, U.K. (e-mail: milutin.jovanovic@unn.ac.uk).

Publisher Item Identifier 10.1109/TEC.2002.801997. ciency (since the rotor losses are much smaller than the BDFIM) and should be able to operate at higher speeds because the cageless rotor can be more robustly constructed. Also, it is easier to model and control than the BDFIM.

BDFRM-based drive technology may find applications in such areas as turbo machinery (where the rugged nature of the rotor and the synchronous machine mode of operation can be exploited), variable-speed constant frequency (VSCF) hydro and wind power applications, commercial heating and air conditioning, as well as in large pump drives.

The tendency of the BDFRM control literature [7]-[11] has been to focus on particular applications or control aspects and not to carry out a complete parameter independent analysis of the machine. The purpose of this paper is to fill this void with a theoretical study of the following BDFRM control properties:

1) conditions for maximum torque per secondary winding amperes;

2) conditions for maximum torque per total primary and secondary winding amperes;

3) power factor control and tradeoffs with inverter voltamperes.

\section{PRELIMinaries}

In the limited space available in this paper, it is not possible to consider the detailed operation and modeling of the BDFRM; see [12] and [13]. This section will, therefore, briefly present some definitions and expressions used in the remainder of this paper.

Fig. 1 shows the basic structure of the BDFRM. As can be seen, the machine logically has two windings wound on the same stator. The primary winding is connected to the grid ${ }^{1}$ and the secondary to an inverter. These two windings always have different pole numbers and, therefore, with a round rotor, there ideally would not be any magnetic coupling between them. However, if the number of salient rotor poles, denoted by $p_{r}$, satisfies the following condition:

$$
p_{r}=p_{p}+p_{s}
$$

where $p_{p}$ and $p_{s}$ are the primary and secondary winding pole pairs, respectively, then there is coupling between the windings. It can be shown that the degree of coupling is dependent on the rotor angle. This implies that there must be a change of co-energy with the rotor movement, and the machine can, con-

\footnotetext{
${ }^{1}$ The fact that the primary winding is connected to a constant voltage and frequency supply means that the primary flux linkage is constant.
} 


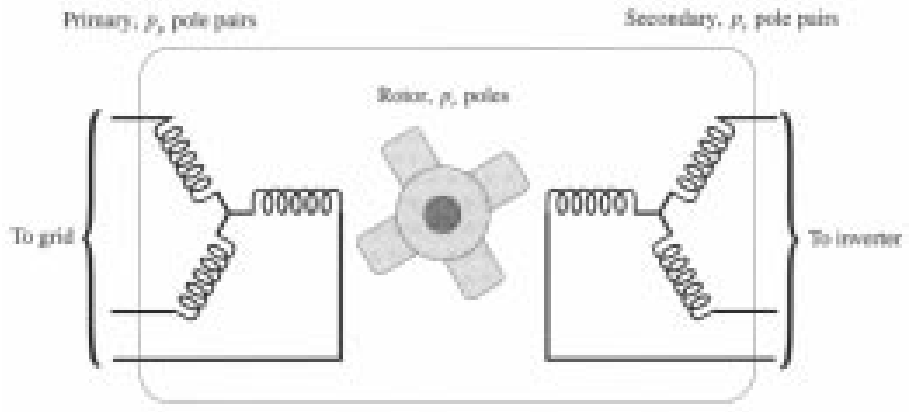

Fig. 1. Conceptual diagram of the BDFRM.

sequently, develop torque. The other key relationship that must exist for a resultant torque to be produced is [12], [13]

$$
\omega_{r}=p_{r} \omega_{r m}=\omega_{p}+\omega_{s}
$$

where $\omega_{r} \triangleq$ the electrical angular velocity of the rotor; $\omega_{r m} \triangleq$ mechanical angular velocity; $\omega_{p} \triangleq$ the supply frequency of the primary winding (radians per second); and $\omega_{s} \triangleq$ the supply frequency of the secondary winding (radians per second).

It is beyond the scope of this paper to develop the complete space vector model of the BDFRM and relevant expressions, in a general reference frame rotating at $\omega$, will simply be stated [9], [12], [13]

$$
\begin{aligned}
& \underline{v}_{p_{r}}=R_{p} \underline{\underline{i}}_{p_{r}}+\frac{d \underline{\lambda}_{p_{r}}}{d t}+j \omega \underline{\lambda}_{p_{r}} \\
& \underline{v}_{s_{r}}=R_{s} \underline{i}_{s_{r}}+\frac{d \underline{\lambda}_{s_{r}}}{d t}+j\left(\omega_{r}-\omega\right) \underline{\lambda}_{s_{r}} \\
& \underline{\lambda}_{p_{r}}=L_{p} \underline{i}_{p_{r}}+L_{p s} \underline{s}_{s_{r}}^{*} \\
& \underline{\lambda}_{s_{r}}=L_{s} \underline{\underline{i}}_{s_{r}}+L_{p s} \underline{i}_{p_{r}}^{*}
\end{aligned}
$$

where $L_{p} \triangleq$ the primary inductance, $L_{s} \triangleq$ the secondary inductance, and $L_{p s} \triangleq$ the primary to secondary mutual inductance. The space vector notation used is as follows: $\underline{x}_{p_{r}}$ and $\underline{x}_{s_{r}}$ denote generic space vectors $\underline{x}$ for the primary and secondary, respectively, in a rotating reference frame.

Remark 1: One of the most distinguishing features of the space vector equations for the BDFRM is that (3) and (4) are in two different reference frames-(3) is in the $\omega$ reference frame, and (4) is in the $\omega_{r}-\omega$ frame.

If one integrates (2), then a similar relationship between the reference frame positions for the primary and secondary windings (denoted by the sub-subscript " $f$ " in Fig. 2) can be obtained:

$$
\theta_{r_{f}}(t)=p_{r} \theta_{r m}(t)=\theta_{p_{f}}(t)+\theta_{s_{f}}(t) .
$$

The angles defined in Fig. 2 are used later in the paper.

Remark 2: The equations for the flux expressions are a little confusing at first sight. Consider (5), for example. This expression appears to contain currents in the primary reference frame (i.e., $\underline{i}_{p_{r}}$ ) as well as currents in the secondary reference frame (i.e., $\underline{i}_{s_{r}}^{*}$ ). However, $\underline{i}_{s_{r}}^{*}$ is a referred secondary current (but in a frequency not turns ratio sense) to the primary reference frame and its components appear as ac quantities of $\omega_{r}-\omega_{s}-\omega=$ $\omega_{p}-\omega$ angular frequency. This is the same frequency as the primary reference frame quantities with respect to the $\omega$ reference

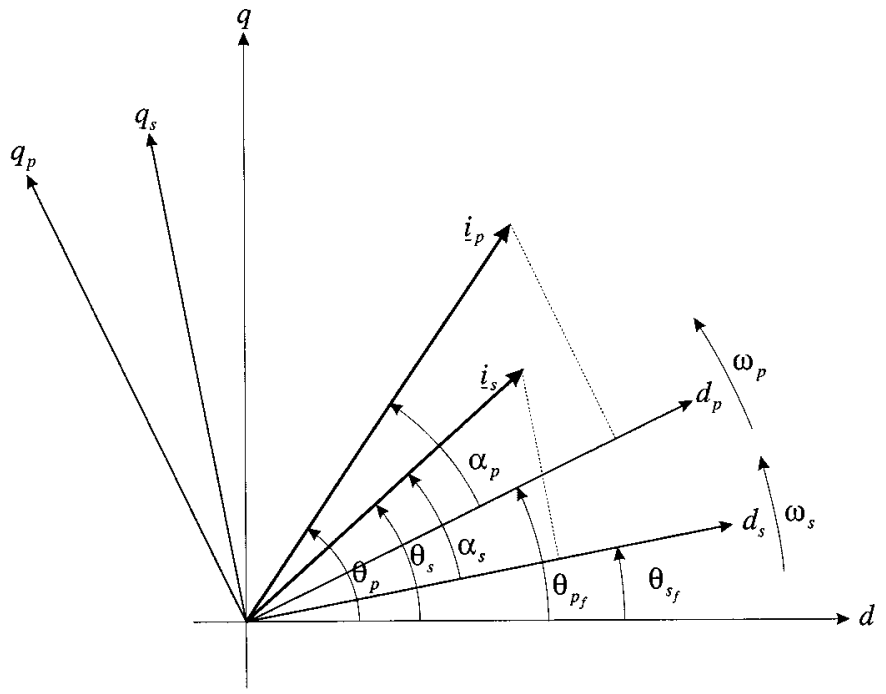

Fig. 2. Reference frames and current angles used for the BDFRM ( $d q$ frame is stationary).

frame. Therefore, the components of the $\underline{i}_{s_{r}}^{*}$ vector are the same as those of the complex conjugate of the secondary current, but they are in the primary flux reference frame. Note, however, that the referred and original current vectors rotate at different velocities with respect to the stationary frame- the referred vector at $\omega_{r}-\omega_{s}=\omega_{p}$ and the original one at $\omega_{s}$. Similarly, $\underline{i}_{p_{r}}^{*}$ in (5) is a referred primary current rotating at $\omega-\omega_{p}$ in the secondary reference frame [12]. The terms $L_{p s} \underline{i}_{s_{r}}^{*}$ and $L_{p s} \underline{i}_{p_{r}}^{*}$ can be interpreted as representing the mutual flux that links the two windings through the modulating action of the rotor. It is this modulating influence of the rotor on the stator magnetomotive forces (MMFs) that results in the previously mentioned frequency conversion.

If we choose the reference frame angular velocity $\omega$ to be the primary frequency, $\omega_{p}$, then given (2), the original voltage (3) and (4) become

$$
\begin{aligned}
& \underline{v}_{p_{r}}=R_{p} \underline{i}_{p_{r}}+\frac{d \underline{\lambda}_{p_{r}}}{d t}+j \omega_{p} \underline{\lambda}_{p_{r}} \\
& \underline{v}_{s_{r}}=R_{s}{\underline{i_{s}}}_{s_{r}}+\frac{d \underline{\lambda}_{s_{r}}}{d t}+j \omega_{s} \underline{\lambda}_{s_{r}} .
\end{aligned}
$$

Using a change of field energy technique, it is possible to derive a number of different expressions for the torque of the BDFRM [12]. The most important one for this paper is

$$
T_{e}=\frac{3}{2} p_{r} \frac{L_{p s}}{L_{p}}\left(\lambda_{p d} i_{s q}+\lambda_{p q} i_{s d}\right)
$$

where the $d q$ components are with respect to the primary and secondary reference frames, the $p$ or $s$ subscript denoting the particular frame.

Remark 3: Equations (8) and (9) are in their "natural" reference frames, with the primary equation in the $\omega_{p}$ frame and the secondary equation in the $\omega_{s}$ frame. Remark 2 still holds in this new reference frame with the exception that the secondary currents are no longer ac but dc and, as a result, are much easier to control. 
Remark 4: Equations (8) and (9) are of the same form as the equations for the DFSRIM. This, at first, is a little surprising due to the different underlying operation principles of the two machines. It should be noted that the dynamics of the BDFRM are not as fast as those of the DFSRIM because of the higher leakage inductances.

Remark 5: Equation (10) is very similar to the $d q$ expression for the torque of the induction machine with rotor flux orientation. This again serves to highlight the close connections between the two machines.

The other main property of the BDFRM that is of interest is its power. The standard space vector power expression [14] has been be used to derive the power-unit (PU) power expressions in Appendix A.

\section{CONTROL PROPERTIES}

The subsequent analysis assumes an ideal machine without saturation. Also, in most cases, the stator resistance is ignored in order to simplify the development of the analytical expressions. This approximation should have little effect on the accuracy of the analysis. For simplicity, the primary and secondary windings are assumed to have the same number of effective turns per pole (i.e., the same inductances $\left(L_{p}=L_{s}\right){ }^{2}$

\section{A. Maximum Torque Per Secondary Ampere}

A desirable property for a BDFRM is to maximize the torque produced for a given inverter current rating [i.e., maximize the torque per secondary ampere (MTPSA)]. Using (10) and aligning the reference frame with the primary flux vector (which effectively means that $\lambda_{p q}=0$ and $\lambda_{p d}=\lambda_{p}$ ), the torque can be written as

$$
T_{e}=\frac{3}{2} p_{r} \frac{L_{p s}}{L_{p}} \lambda_{p} i_{s q} .
$$

Remark 6: The primary flux frame alignment used in (11) makes it obvious that one can control the torque in the BDFRM independently from the flux. $\lambda_{p}$ is fixed by the grid supply voltage and frequency is applied to the primary winding and the torque is controlled by the secondary winding $q$ axis current $\left(i_{s q}\right)$.

From Fig. 2, one can see that $i_{s q}=i_{s} \sin \alpha_{s}$, and consequently, the torque per secondary ampere can be written as

$$
\frac{T_{e}}{i_{s}}=\frac{3}{2} p_{r} \frac{L_{p s}}{L_{p}} \lambda_{p} \sin \alpha_{s}
$$

which is clearly a maximum when $\alpha_{s}=\pi / 2$.

Remark 7: Examination of (12) indicates that the torque per secondary ampere improves with increasing the $L_{p s} / L_{p}$ ratio. It can be shown that this implies a larger saliency ratio for the rotor [15]. Therefore, the machine should be designed with the highest possible rotor saliency. The use of an axially laminated rotor would be a preferable solution from this point of view, but it would also have high iron losses and be costly and difficult to manufacture. Recent radial laminated flux barrier rotor designs have nearly the same saliency ratio of the axially laminated design, are economic to manufacture, and have low iron losses [16], [17].

\footnotetext{
${ }^{2}$ This choice of inductance may not be optimal when it comes to obtaining the maximum torque for a given amount of active copper and iron in the machine.
}

Remark 8: Note that this control strategy only minimizes the secondary current-no consideration has been given to the current or the power factor of the grid connected (primary) winding.

\section{B. Maximum Torque Per Total Amperes}

An alternative strategy for controlling the BDFRM may be the maximum torque per total amperes (MTPTA) of the machine. The total amperes refers to the sum of the primary and secondary current magnitudes. In other words, this strategy is essentially trying to share the current more evenly between the windings for a given torque output.

The problem to be solved can be stated mathematically in normalized terms as ${ }^{3}$

$$
\min _{\alpha_{s}, \alpha_{p}} i_{t n}=i_{p n}+i_{s n}=\frac{\zeta \sin \alpha_{p}+\sin \alpha_{s}}{\sin \left(\alpha_{s}+\alpha_{p}\right)}
$$

subject to a specific torque output $T_{n}$.

Remark 9: The $\alpha_{s}$ and $\alpha_{p}$ variables in (13) are not independent due to the primary flux frame alignment condition implicit in (13) [18].

Equation (13) can, after considerable manipulation, be put into the following form [18]:

$i_{t n}=\frac{\zeta T_{n} \sqrt{1+\tan ^{2} \alpha_{s}}+\sqrt{\left(2 \tan \alpha_{s}-T_{n}\right)^{2}+T_{n}^{2} \tan ^{2} \alpha_{s}}}{2 \tan \alpha_{s}}$.

Theoretically, one can differentiate (14) with respect to $\tan \alpha_{s}$ and then equate the obtained expression to zero to solve for the $\tan \alpha_{s}$ that will minimize $i_{t n}$. However, in practice, the resultant derivative expression is very complex and cannot be solved analytically. Therefore, the optimal angles have been calculated using a numerical minimization procedure.

The plots developed from (14) are for the case where $L_{p}=$ $L_{s}$ and the rotor has an equivalent synchronous reluctance machine saliency ratio of $\xi=L_{d} / L_{q}=8$, which can be shown to be equivalent to $\zeta=9 / 7$ [15], [18]. The plots are all developed for $\omega_{s n}=1$ and torque levels ranging from 0.1 to $1.5 \mathrm{PU}$

Fig. 3 shows the current angles required to achieve MTPTA operation.

Remark 10: One interesting feature of Fig. 3 is that $\alpha_{s}$ does not change as much as $\alpha_{p}$. Also, the $\alpha_{s}$ angles are larger compared to $\alpha_{p}$ for all the torque levels. This result is expected as the primary winding and is mainly responsible for the machine magnetization as opposed to the secondary which is torque producing. One can see from the same figure that at the low torques, the primary angle approaches zero, indicating that it is supplying almost all of the flux for the machine. The angle of the secondary current in this torque region, at first, would seem to indicate that it is also supplying significant flux to the machine. However, as we shall see in a subsequent plot, the magnitude of $i_{s n}$ approaches zero under these conditions. One could anticipate that this would be the case from (12).

Remark 11: The values of the individual winding current magnitudes can be found from (20) and (21). Note that $i_{p m}$ and $i_{s n}$ do not explicitly contain the torque level $T_{n}$. However, as $T_{n}$ varies, the $\alpha_{s}$ and $\alpha_{p}$ vary as illustrated in Fig. 3 .

It would be instructive to break down the total current magnitude into the primary and secondary components under this condition. Fig. 4 shows the individual current magnitudes.

\footnotetext{
${ }^{3} \zeta$ is defined in Appendix A.
} 


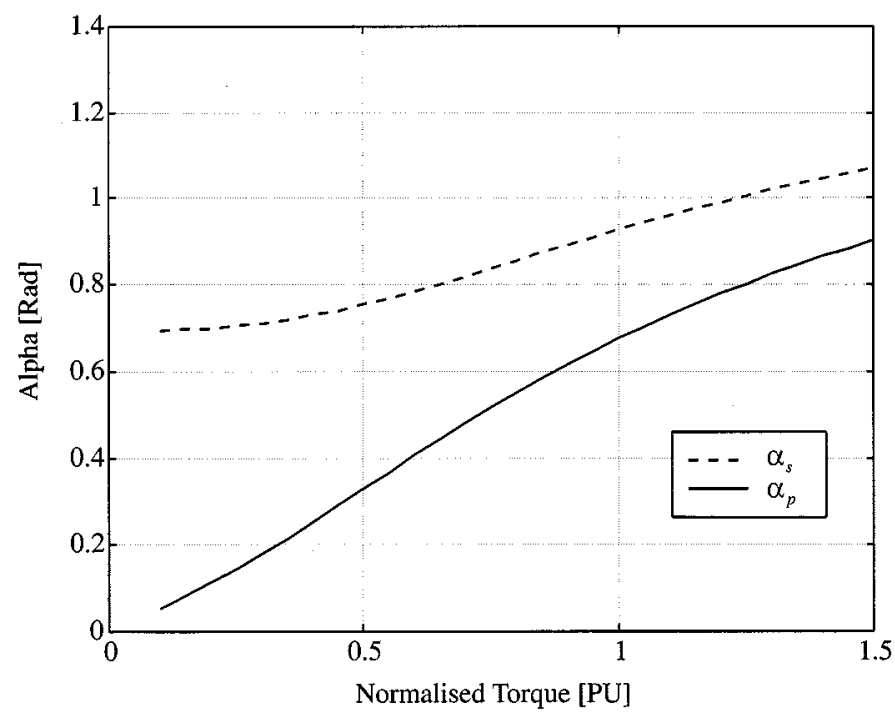

Fig. 3. $\alpha_{s}$ and $\alpha_{p}$ angles for maximum torque per total amperes.

Remark 12: Fig. 4 demonstrates that the change in the total current magnitude, with respect to torque, largely results from the variation in the magnitude of the secondary current component. This is not unexpected, since from (11) one can see that the torque is directly related to the secondary $q$ axis current. From Fig. 3, it is evident that $\alpha_{s}$ does not change a lot with torque. Therefore, the $i_{s n}$ must be changing to affect the increased $i_{s q n}$ component and, hence, torque output [see (19)].

Remark 13: The other relevant remark about Fig. 4 is that the primary current magnitude is relatively constant with torque variations. From Fig. 3, one can see that $\alpha_{p}$ increases considerably with increased torque. Therefore, one would conclude that the primary current contributes less to the primary flux with rising torque levels, as opposed to the secondary current which increases its contribution to the flux (since $\lambda_{p}$ is constant).

The statements in Remark 13 lead naturally to consideration of the power components for the primary and secondary windings. Fig. 5 shows these components under the MTPTA condition, developed using (24)-(27).

Remark 14: Examination of Fig. 5 sheds extra light on the comments in Remarks 12 and 13. One important observation is that the real power contributed by the primary and secondary windings are the same. This is a consequence of the $\omega_{s n}=1$ assumption as follows from (24) and (26). If $\omega_{s n}<1$, then the power contributed by the secondary winding becomes less. At the extreme, if $\omega_{s n}=0$ (i.e., dc) then the secondary power is zero, since the secondary winding is now simulating the field winding of a classical synchronous machine. ${ }^{4}$

Remark 15: The reactive powers in Fig. 5 show that the primary reactive power decreases with increased torque. This indicates that the primary winding participates less in the flux production (as noted earlier). On the other hand, one can see that the opposite occurs for the secondary winding, with the corresponding reactive power increasing significantly with increased torque.

${ }^{4}$ Note that under this condition, there will be enough real power to supply the resistive losses of the winding. This does not show up in the analysis since we are neglecting resistances.

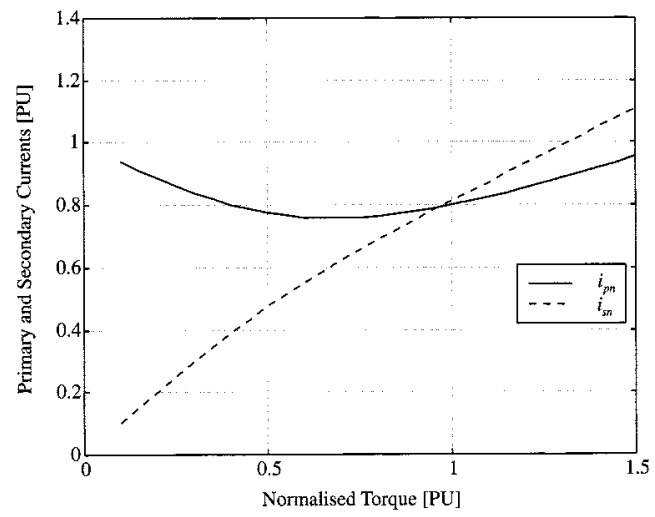

Fig. 4. Current magnitude components under maximum torque per total amperes.

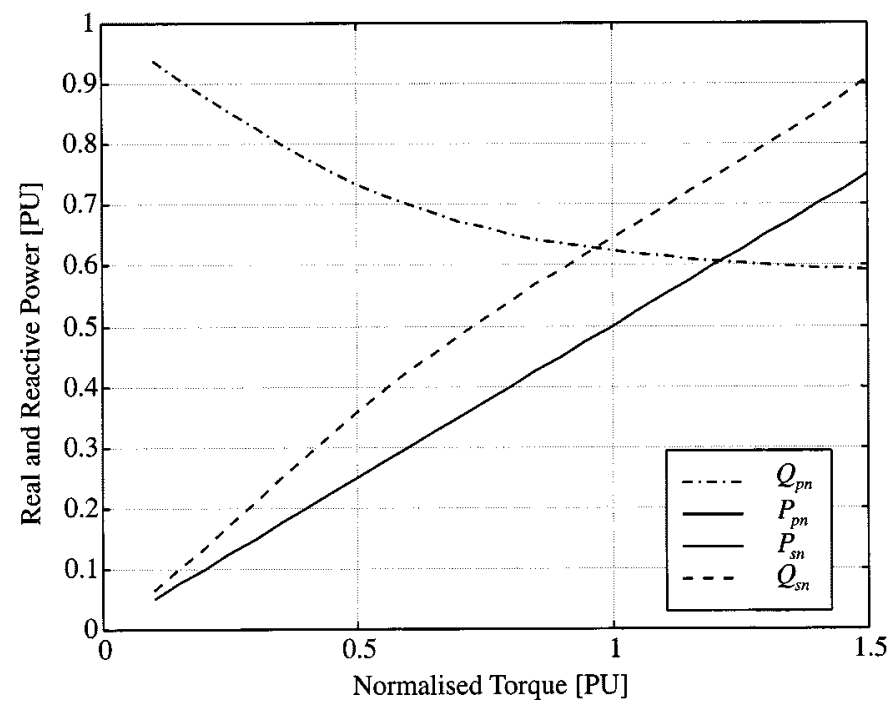

Fig. 5. Real and reactive powers under the maximum torque per total amperes condition with the secondary winding being supplied at the mains frequency (note that the real power of the windings is the same as $\omega_{s n}=1$ ).

\section{Power, Power Factor, and Volt-Amperes}

In order to understand the power relationships for the BDFRM, we shall look at the power performance of the machine under constant torque and constant secondary frequency.

Consider (19), which we can rearrange to give

$$
F\left(\alpha_{p}, \alpha_{s}\right)=2 \sin \alpha_{s} \sin \alpha_{p}-T_{n} \sin \left(\alpha_{p}+\alpha_{s}\right)=0 .
$$

If we choose a value of $\alpha_{s}$ in (15) then we can solve for $\alpha_{p}$ for a specific value of $T_{n}$. In the following analysis, we shall assume that the torque level is $T_{n}=1$, and the secondary frequency is $\omega_{s n}=1$. At the end of this section, we shall briefly discuss what happens to the results as the torque and frequency are varied from these values.

The $\alpha_{p}$ and $\alpha_{s}$ profile resulting from (15) can be used to generate the magnitudes of the primary and secondary currents. This analysis gives one a good feel for the tradeoffs that occur in relation to the inverter size to achieve particular power factor outcomes. Using (20) and (21), one can develop the plots shown in Fig. 6. 


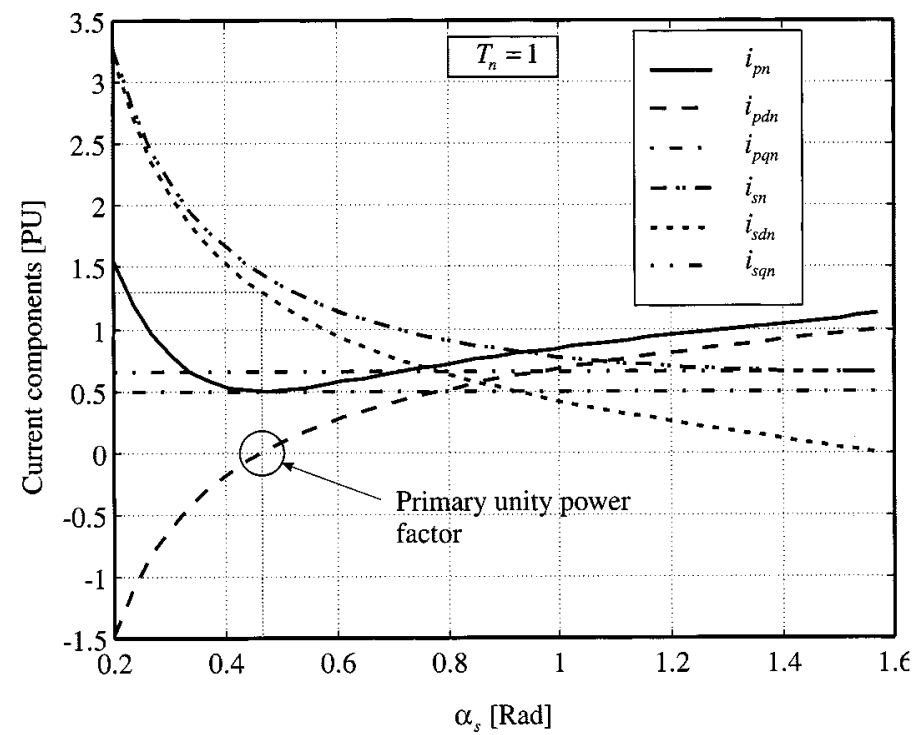

Fig. 6. Primary and secondary currents, $T_{n}=1$, and $\omega_{s n}=1$.

Examination of Fig. 6 immediately reveals that $i_{m}$ is at a minimum when $\alpha_{s} \approx 0.46 \mathrm{rad}$. This point corresponds to the angle when the secondary winding is developing all of the primary flux for the machine (through the mutually coupled flux), which, in turn, implies the $\alpha_{p}=\pi / 2$. This means that the primary current is orthogonal to $\lambda_{p}$ and, hence, the primary current by itself does not produce any flux. One can see from Fig. 6 that the $i_{p d n}$ component of the current is zero at this angle, further confirming the analysis. The other relevant aspect of the figure is that $i_{p q n}$ is constant over the whole range of $\alpha_{s}$ angles. This is the component of the primary current that is in quadrature with $\lambda_{p}$ and, therefore, is the torque producing component (i.e., the coupled secondary) $q$ axis current [18]. Given that $\omega_{p m}$ is fixed by the winding grid connection, the real power contributed by the primary is constant too.

Fig. 6 also shows the secondary current components. The most significant feature is the very high secondary current magnitude for low values of $\alpha_{s}$. One can see that most of the increase in $i_{s n}$ is due to the increase of the flux producing $i_{s d n}$ component. For very small angles of $\alpha_{s}$, the machine can become overexcited and the primary winding begins to look capacitive. Note that, similar to the primary winding, the $q$ axis component $\left(i_{s q n}\right)$ of the current remains constant over the whole angle range.

Fig. 7 shows the power components under the same condition as Fig. 6.

Remark 16: In Fig. 7, note that the power factor of the primary is unity when $\alpha_{s}=0.46 \mathrm{rad}$ and that the real power component of the primary winding is constant.

Remark 17: Fig. 7 graphically shows the significant change in the reactive power supplied by the secondary winding when $\alpha_{s}<0.46$ radian. As $\alpha_{s} \rightarrow \pi / 2$, then the reactive power of the secondary also approaches zero but does not reach zero at $\alpha_{s}=\pi / 2$. This is because the secondary reference frame is out of alignment with the secondary flux. Therefore, there is still a secondary flux producing component of current with $i_{s d n}=0$.

One of the most interesting properties of the BDFRM is the ability to control the power factor of the primary via the sec-

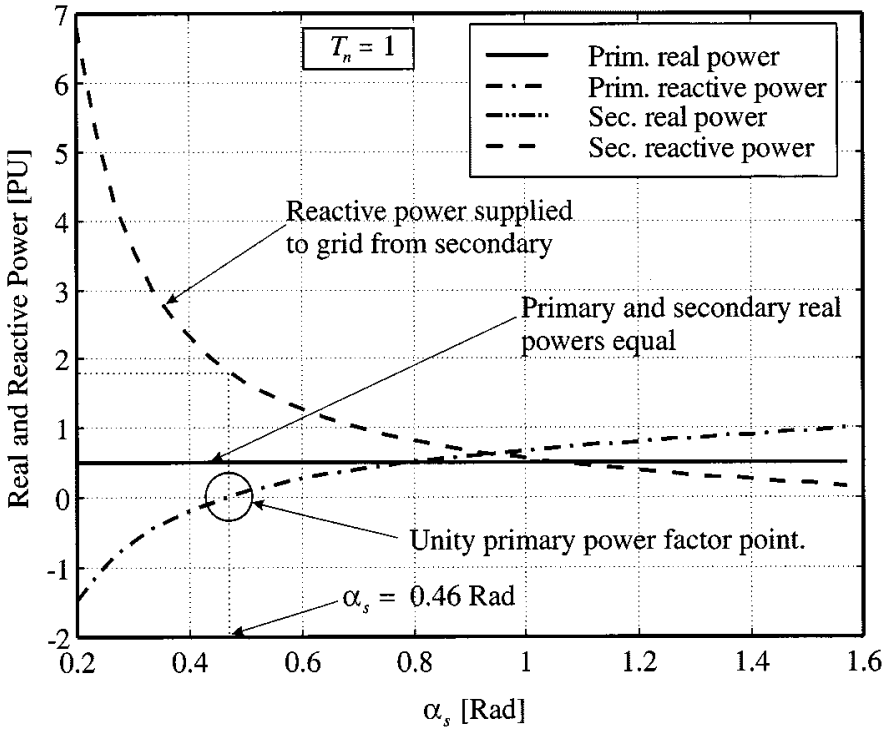

Fig. 7. Primary and secondary real and reactive powers, $T_{n}=1$, and $\omega_{s n}=$ 1

ondary winding currents. Fig. 8 shows the primary power factor. This plot shows that the power factor of the primary does indeed become zero at $\alpha_{s}=0.46$ radian. If one plotted the secondary power factor, one would find that it is very poor for $\alpha_{s} \approx 0.3$, monotonically increasing with $\alpha_{s}$, and nearly reaching one at $\alpha_{s}=\pi / 2$.

The comments in the previous paragraph are further reinforced if one examines the volt-amperes (VA) absorbed by the secondary windings under the $T_{n}=\omega_{s n}=1$ condition. Fig. 9 shows the secondary VA.

The discussion thus far has concentrated on the tradeoffs associated with the control of the primary imaginary (or reactive) power. However, in the power plots, one can notice that the real power delivered by the primary and secondary are equal (under the $\omega_{s n}=1$ condition). If one examines (26), one can see that for a given nonzero torque, the real power delivered by the secondary is related to the applied secondary frequency, whereas the real power delivered by the primary is independent of the secondary frequency [see (24)]. If $\omega_{s n}>1$ in (26) (i.e., high-speed operation) then the real power contributed by the secondary will be greater than that of the primary, otherwise (for $\omega_{s n}<1$ ) it is less. Clearly, if $\omega_{s n}=0$ then the secondary power is zero and the BDFRM operates as a synchronous machine.

If the phase sequence is reversed, then $\omega_{s n}<0$. This allows the machine to operate at subsynchronous speeds (i.e., at $\omega_{r m}<\omega_{p} / p_{r}$ ). If one considers (26) one can see that negative $\omega_{s n}$ corresponds to a negative $P_{s n}$. Therefore, power is being regenerated from the secondary back into the supply. Note that in this mode, the inverter must be capable of regeneration for sustained operation. When operating at speeds below synchronous speed, the machine is very inefficient since energy is being taken from the supply through the primary and then returned to the supply via the secondary. Therefore, some of the input energy is circulating from the primary to the secondary, incurring losses along the way. 


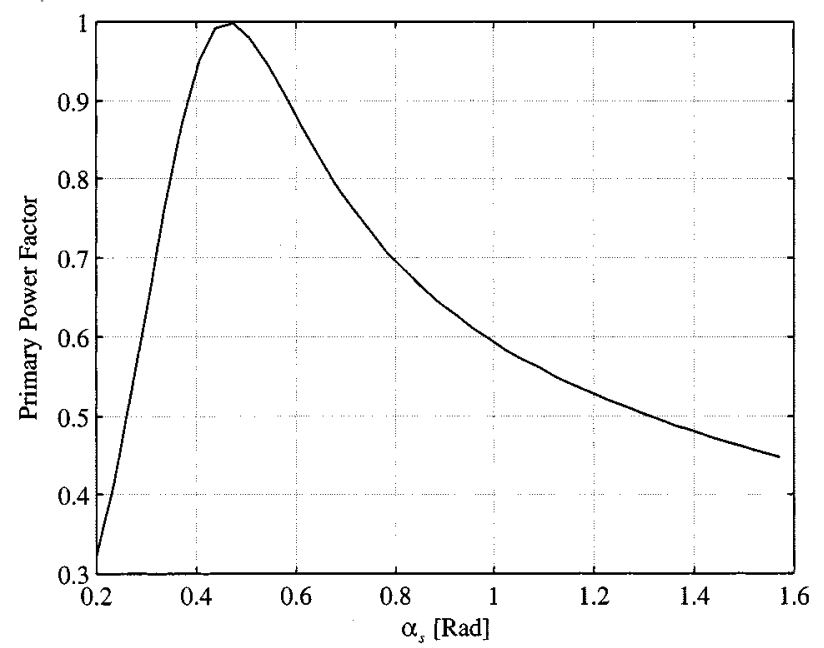

Fig. 8. Primary winding power factor when $T_{n}=1$ and $\omega_{s n}=1$.

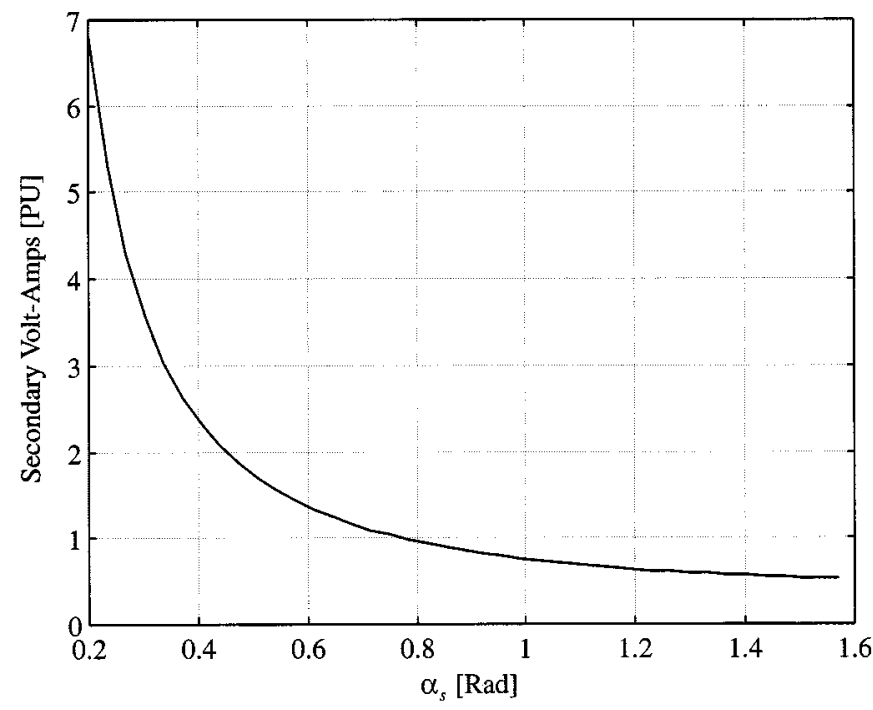

Fig. 9. Secondary winding VA with $T_{n}=1$ and $\omega_{s n}=1$.

All of the previous plots were for the case of $T_{n}=1$ and $\omega_{s n}=1$. If these parameters are varied, then the following further observations can be made. For the case of varying $\omega_{s n}$, we have the following.

- The real and imaginary current magnitudes flowing in the windings do not change.

- The current angle profile remains the same.

- As a consequence of the first two comments, the maximum torque per total amperes does not vary.

- The primary real and imaginary powers are secondary frequency independent and, therefore, are unaffected by its variations.

- The secondary real and imaginary powers and VA decrease at all $\alpha_{s}$ angles in proportion to $\omega_{s n}$.

- Under the $\omega_{s n}=0$ condition, the $\alpha_{s}$ angle effectively becomes the torque angle of a synchronous machine. It corresponds to the initial phase of the secondary current relative to the secondary reference frame (which is now stationary). The latter is initially aligned with the high permeance axis of the rotor.
If the torque level is varied at $\omega_{s n}=1$, then the following observations can be made.

- The primary and secondary current angles approach zero as $T_{n} \rightarrow 0$.

- The real power is still evenly shared between the primary and secondary windings.

- The $\alpha_{s}$ versus $\alpha_{p}$ current angle profile changes with variations in $T_{n}$.

- The optimal torque per total amperes change, although only by a small amount.

\section{Control Scheme}

The BDFRM can be configured into a drive system, employing vector control techniques [10] and [11]. In case of primary flux orientation discussed previously, then a drive control system of the form of Fig. 10 can be devised. ${ }^{5}$ As the secondary winding quantities are only controllable, the secondary reference frame position $\theta_{s_{f}}$ should be determined for control implementation. This can be achieved using the primary frame position $\theta_{p_{f}}$ (which is the same as the primary flux angle due to the alignment condition and can, therefore, be estimated from the measured grid voltages ${ }^{6}$ ), rotor position measurement $\theta_{r m_{f}}$, and (7). The desired inputs to the $d q$ current controllers depend on a control strategy to be implemented. For example, if the maximum torque per secondary ampere strategy is of interest, then $i_{s d}^{*}$ and $i_{s q}^{*}$ are determined from (11) for a given torque command $T_{e}^{*}$.

\section{ConClusions}

This paper has presented a number of properties of the BDFRM. The main feature of the machine is that it allows a smaller inverter to be used if the output speed range required is relatively small. However, as has been shown in the analysis before, if one uses the ability of the inverter to control the primary winding power factor, then the inverter size has to increase, the amount depending on the power factor one wishes to obtain.

In addition to the machine attributes examined, the paper has also introduced a number of PU-based model equations for the BDFRM.

To summarize, the main conclusions that can be drawn from the paper are the following.

1) The maximum torque per secondary ampere (MTPSA) is achieved if $\alpha_{s}=\pi / 2$. This angle is independent of the applied secondary frequency and the torque level.

2) If the machine is operated with $\alpha_{s}=\pi / 2$, the primary power factor is poor (i.e., $\approx 0.47$ ). In many applications this may not be satisfactory.

3) The MTPTA current angles for the BDFRM do not equal the MTPSA current angle. Furthermore, the MTPTA angles depend on the torque level at which the machine is operating.

${ }^{5}$ Fig. 10 does not show the circuitry required for starting the system. If a partially rated inverter is used, then an auxiliary contactor is usually required to short the secondary. This allows the machine to start as an induction machine. Once the machine is near the synchronous speed, the contactors are opened, and the inverter is connected.

${ }^{6}$ Estimation errors can occur by ignoring the primary winding resistance, but in most cases, this error is negligible because of a fixed $50-\mathrm{Hz}$ supply. 


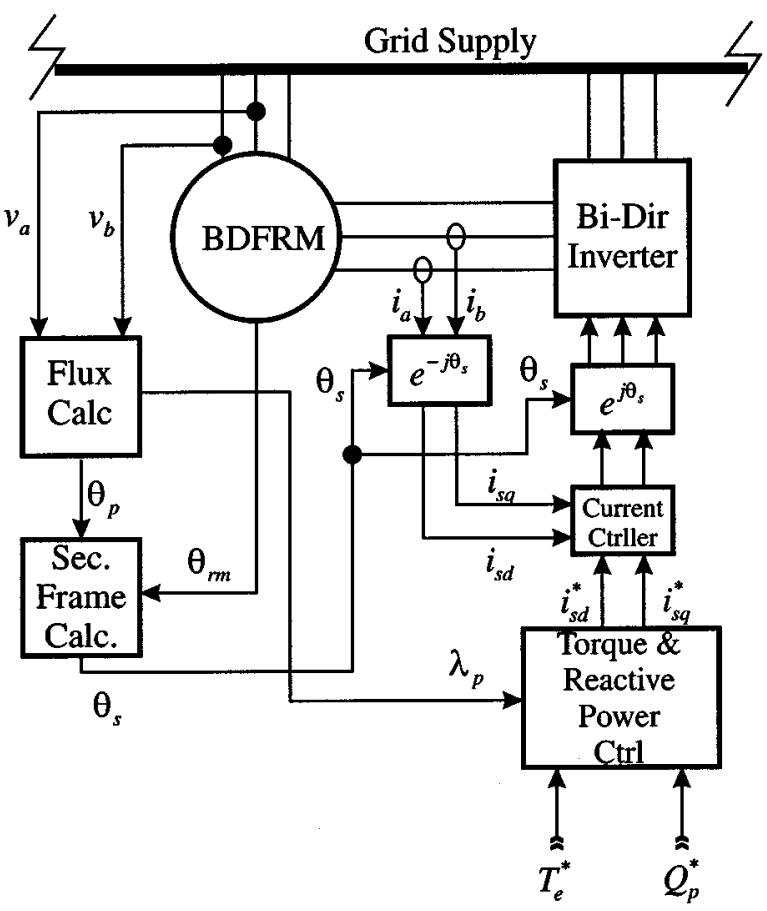

Fig. 10. Block diagram of a BDFRM-based drive system.

4) The paper has highlighted the implications of primary power factor control on the rating of the secondary winding inverter.

The analysis in this paper, due to space restrictions, is necessarily limited. Nevertheless, it indicates that the BDFRM has many interesting properties that can be potentially used in many applications. The authors believe that the BDFRM deserves more investigation to determine whether it is industrially viable in the target application.

\section{APPENDIX}

\section{A. Normalized Parameters}

It is possible to derive a set of PU bases for the BDFRM corresponding to an arbitrarily chosen $\alpha_{s}=\alpha_{p}=\pi / 4$ current angle condition [18]

$$
\begin{aligned}
T_{B}=\frac{3}{4} p_{r} \frac{\lambda_{p}^{2}}{L_{p}} ; & P_{B}=\frac{2 \omega_{B}}{p_{r}} T_{B} \\
i_{B}=\frac{\lambda_{B}}{L_{B}}=\frac{\lambda_{p}}{L_{p}} ; & \lambda_{B}=\frac{v_{B}}{\omega_{B}}=\lambda_{p} \\
\omega_{B}=\omega_{p}=2 \pi f_{B} ; & L_{B}=L_{p}
\end{aligned}
$$

where $v_{B}$ and $f_{B}$ are the grid supply voltage and frequency.

These base values can be used to convert the main performance equations into the normalized forms that appear in (23)

$$
\begin{aligned}
& \text { Torque: } T_{n}=\frac{2 \sin \alpha_{s} \sin \alpha_{p}}{\sin \left(\alpha_{p}+\alpha_{s}\right)} \\
& =\frac{2}{\zeta} i_{s n} \sin \alpha_{s} \\
& \text { Primary current: } i_{p n}=\frac{\sin \alpha_{s}}{\sin \left(\alpha_{p}+\alpha_{s}\right)}
\end{aligned}
$$

$$
\begin{aligned}
\text { Secondary current: } i_{s n} & =\frac{\zeta \sin \alpha_{p}}{\sin \left(\alpha_{p}+\alpha_{s}\right)} \\
\text { Primary flux: } \lambda_{p n} & =1 \\
\text { Secondary flux: } \underline{\lambda}_{s n} & =L_{s n} \underline{i}_{s n}+\frac{1}{\zeta} \underline{i}_{m}^{*}
\end{aligned}
$$

The PU real and reactive power expressions are

$$
\begin{aligned}
P_{p n}= & \frac{1}{\zeta} i_{s n} i_{p n} \sin \left(\alpha_{s}+\alpha_{p}\right) \\
= & \frac{\sin \alpha_{p} \sin \alpha_{s}}{\sin \left(\alpha_{s}+\alpha_{p}\right)}=\frac{1}{2} T_{n} \\
Q_{p n}= & 1-\frac{i_{s d n}}{\zeta}=\frac{\sin \alpha_{s} \cos \alpha_{p}}{\sin \left(\alpha_{s}+\alpha_{p}\right)} \\
P_{s n}= & \frac{1}{\zeta} \omega_{s n} i_{s n} i_{m n} \sin \left(\alpha_{s}+\alpha_{p}\right)=\frac{\omega_{s n}}{2} T_{n} \\
Q_{s n}= & \frac{\omega_{s n} \sin \alpha_{p}}{\sin ^{2}\left(\alpha_{s}+\alpha_{p}\right)} \\
& \cdot\left[\frac{\sin \alpha_{p}}{k_{p s}^{2}}+\sin \alpha_{s} \cos \left(\alpha_{s}+\alpha_{p}\right)\right]
\end{aligned}
$$

where $\zeta=L_{p} / L_{p s}$ and $k_{p s}=L_{p s} / \sqrt{L_{p} L_{s}}$. The " $n$ " subscripts denote that the relevant quantity is a PU or normalized quantity.

\section{REFERENCES}

[1] L. Hunt, "A new type of induction motor," J. Inst. Elect. Eng., vol. 38, pp. 648-667, 1907.

[2] 1 , "The cascade induction motor," J. Inst. Elect. Eng., vol. 52, pp. 406-426, 1914.

[3] F. Creedy, "Some developments in multi-speed cascade induction machines," J. Inst. Elect. Eng., vol. 59, pp. 511-532, 1921.

[4] A. Broadway and L. Burbridge, "Self-cascaded machine: A low speed motor or a high frequency brushless alternator," Proc. Inst. Elect. Eng., vol. 117, pp. 1277-1290, July 1970.

[5] A. Broadway, "Cageless induction machines," Proc. Inst. Elect. Eng., vol. 118, pp. 1593-1600, Nov. 1971.

[6] A. Broadway, B. Cook, and P. Neal, "Brushless cascade alternator," Proc. Inst. Elect. Eng., vol. 121, pp. 1529-1535, Dec. 1974.

[7] L. Xu, F. Liang, and T. Lipo, "Transient model of a doubly excited reluctance motor," IEEE Trans. Energy Conversion, vol. 6, pp. 126-133, Mar. 1991.

[8] L. Xu and Y. Tang, "A novel wind-power generating system using field orientation controlled doubly-excited brushless reluctance machine," in Proc. IEEE IAS Annu. Meet., 1992, pp. 408-418.

[9] Y. Liao, L. Zhen, and L. Xu, "Design of a doubly-fed reluctance motor for adjustable speed drives," in Proc. IAS Annu. Meet., vol. 1, Oct. 1994, pp. $305-312$.

[10] Y. Tang and L. Xu, "Vector control and fuzzy logic control of doubly fed variable speed drives with DSP implementation," IEEE Trans. Energy Conversion, vol. 10, pp. 661-668, Dec. 1995.

[11] L. Xu, L. Zhen, and E. Kim, "Field-orientation control of a doubly excited brushless reluctance machine," IEEE Trans. Ind. Applicat., vol. 34, pp. 148-155, Jan./Feb. 1998.

[12] R. Betz and M. Jovanović. (1998) Introduction to Brushless Doubly Fed Reluctance Machines-The Basic Equations. Dept. Elec. Energy Conversion, Aalborg University, Aalborg, Denmark. [Online]. Available: ftp://murray.newcastle.edu.au/pub/reb/Papers/BDFRMRev.pdf

[13] F. Liang, L. Xu, and T. Lipo, "D-q analysis of a variable speed doubly AC excited reluctance motor," Elect. Machines Power Syst., vol. 19, pp. 125-138, Mar. 1991.

[14] P. Vas, Vector Control of AC Machines. Oxford, U.K.: Oxford Univ. Press, 1990.

[15] R. Betz and M. Jovanović, "The brushless doubly fed reluctance machine and the synchronous reluctance machine-A comparison," in Proc. IAS Annu. Meet., Oct. 1999.

[16] A. Vagati and T. Lipo, "Synchronous reluctance motors and drives-A new alternative," IEEE Ind. Applicat. Soc., A. Vagati and T. Lipo, Eds., 1994. 
[17] A. Vagati, A. Canova, M. Chiampi, M. Pastorelli, and M. Repetto, "Improvement of synchronous reluctance motor design through finite-element analysis," in Conf. Rec. 34th IEEE Ind. Applicat. Soc. Annu. Meet., vol. 2, Oct. 1999, pp. 862-871.

[18] R. Betz and M. Jovanović. (1998) Comparison of the brushless doubly fed reluctance machine and the synchronous reluctance machine. Dept. Elect. Energy Conversion, Inst. Energy Technol., Aalborg University, Aalborg, Denmark. [Online]. Available: ftp://murray.newcastle.edu.au/pub/reb/Papers/bdfrm-sync.pdf

Robert E. Betz (M'92) received the B.E., M.E. and Ph.D. degrees from the University of Newcastle, Callaghan, Australia, in 1979, 1982, and 1984, respectively.

He is currently an Associate Professor in the Department of Electrical and Computer Engineering, University of Newcastle. His major interests are electrical machine drives, real-time operating systems, and industrial electronics.

Dr. Betz is a member of the Industrial Drives nd Electric Machines Committees of the IEEE Industry Applications Society.
Milutin G. Jovanović (M'99) received the Dipl.Eng and M.E.E. degrees from the University of Belgrade, Belgrade, Yugoslavia, in 1987 and 1991, respectively, and the Ph.D. degree from the University of Newcastle, Callaghan, Australia, in 1997.

He is currently a Senior Lecturer in the School of Engineering, University of Northumbria, Newcastle, U.K. His main interests are in the areas of electrical machines and drives, power electronics, and power systems.

Dr. Jovanović is a member of the Electric Machines and Industrial Drives Committees of the IEEE Industry Applications Society. 